\title{
A Trade-Off Between Collision Probability and Key Size in Universal Hashing Using Polynomials
}

\author{
Palash Sarkar \\ Applied Statistics Unit \\ Indian Statistical Institute \\ 203, B.T. Road, Kolkata \\ India 700108. \\ email: palash@isical.ac.in
}

\begin{abstract}
Let $\mathbb{F}$ be a finite field and suppose that a single element of $\mathbb{F}$ is used as an authenticator (or tag). Further, suppose that any message consists of at most $L$ elements of $\mathbb{F}$. For this setting, usual polynomial based universal hashing achieves a collision bound of $(L-1) /|\mathbb{F}|$ using a single element of IF as the key. The well-known multi-linear hashing achieves a collision bound of $1 /|\mathbb{F}|$ using $L$ elements of $\mathbb{F}$ as the key. In this work, we present a new universal hash function which achieves a collision bound of $m\left\lceil\log _{m} L\right\rceil /|\mathbb{F}|, m \geq 2$, using $1+\left\lceil\log _{m} L\right\rceil$ elements of $\mathbb{F}$ as the key. This provides a new trade-off between key size and collision probability for universal hash functions.

Keywords: universal hash function, polynomial based hashing.
\end{abstract}

\section{Introduction}

Universal hash functions are of fundamental importance in cryptography and computer science [13, 5]. Being of practical importance, there have been efforts $[7,11,4,1]$ to design hash functions which are very fast.

Polynomial based universal hashing over a finite field $\mathbb{F}$ has a collision bound of $(L-1) /|\mathbb{F}|$ for hashing messages consisting of $L$ elements of $\mathbb{F}$. Both the authenticator (or tag) and the key are single elements of $\mathbb{F}$ and computing the digest requires $L-1$ multiplications over $\mathbb{F}$. Bernstein [2] defined a new class of polynomials for which the number of multiplications can be halved at the negligible cost of increasing the collision bound to $(2 L-1) /|\mathbb{F}|$. The authenticator and the key are still single elements of $\mathbb{F}$. In contrast, for the well-known multi-linear hash function [6,5], the collision bound is $1 /|\mathbb{F}|$ at the cost of making the key length equal to that of the message. The question that we ask is whether there is some intermediate trade-off?

To this end, we first propose a general construction of universal hash function. This construction builds a hash function working on arbitrary length strings from hash functions working on fixed size domains. In this sense, the new construction can be considered to be a domain extender for universal hash function. The basic idea is to take mutually independent hash functions $f_{1}, \ldots, f_{\ell}$ and combine them using a multi-level construction which uses one hash function at each level. Hashing messages with variable lengths requires an additional subtlety which is taken care of by using another XOR universal random function $\psi$.

Instantiating the functions $f_{i}$ using usual polynomial hashing gives rise to a multi-variate polynomial. The collision bound is $m\left\lceil\log _{m} L\right\rceil /|\mathbb{F}|$, where $L$ is as before the maximum number of elements of $\mathbb{F}$ in any message and $m$ can be any value $\geq 2$. The number of multiplications required to compute the tag remains the same as that required for polynomial hashing. The trade-off is that the key consists of $1+\left\lceil\log _{m} L\right\rceil$ elements of $\mathbb{F}$. For 128-bit tags corresponding to $\mathbb{F}=G F\left(2^{n}\right)$, 
Table 1 compares the collision probabilities and key sizes of the above mentioned hashing methods. For a collision bound of $2^{-96}$, polynomial hashing is better since it requires a smaller key. On the other hand, with the new hashing method, using keys consisting of 3, 5,9 and 17 elements, it is possible to progressively reduce the collision probabilities. This underlines the new trade-offs that are made possible by the new method.

Table 1. Comparative study of collision bounds and key sizes of different hashing methods for 128-bit tags and $L=2^{32}$. The key size is given in terms of the number of elements of $G F\left(2^{128}\right)$ that is required.

\begin{tabular}{|l|c|c|}
\hline method & coll. bnd. & key sz. \\
\hline poly hash & $2^{-96}$ & 1 \\
\hline multi-linear hash & $2^{-128}$ & $2^{32}$ \\
\hline \hline new hash with $m=2^{2}$ & $2^{-122}$ & 17 \\
\hline new hash with $m=2^{4}$ & $2^{-121}$ & 9 \\
\hline new hash with $m=2^{8}$ & $2^{-108}$ & 5 \\
\hline new hash with $m=2^{16}$ & $2^{-111}$ & 3 \\
\hline new hash with $m=2^{32}$ & $2^{-96}$ & 2 \\
\hline
\end{tabular}

The basic multi-level construction can also be combined with the class of polynomials due to Bernstein [2] based on earlier work due to Rabin and Winograd [8], which we call the BRW polynomials. More interestingly, it is possible to instantiate some of the $f_{i}$ 's using BRW polynomials and the others using usual polynomials.

Prior and related works. Universal hash functions were introduced in [5] and have been extensively studied since then. See for example $[13,3,12,9]$.

Using results from Stinson [12], it can be shown that if $f_{1}$ and $f_{2}$ have collision bounds $\varepsilon_{1}$ and $\varepsilon_{2}$ respectively, then the function $f_{2}\left(f_{1}\left(\mathbf{x}_{1}, \ldots, \mathbf{x}_{k}\right)\right)$ has a collision bound $\varepsilon_{1}+\varepsilon_{2}$. We develop this idea further into a multi-level hashing algorithm which can handle variable and arbitrary length messages.

The basic idea of our construction is to format the message into elements of $\mathbb{F}$ and then arrange the elements themselves into groups. Applying $f_{1}$ to each group again provides a sequence of elements of $\mathbb{F}$ which are again arranged into groups and $f_{2}$ is applied to each group. The process is continued until we end with a single element of $\mathbb{F}$. Analysis is relatively straightforward when messages consists of a fixed number of elements of $\mathbb{F}$. Difficulty occurs when we are interested in hashing variable length bit strings. For one thing, we need to consider conversion from elements of $\mathbb{F}$ to bit strings and vice versa. More subtle, however, is the requirement of handling length variability and partial blocks. We believe that the new construction of the complete hash function is a non-trivial extension of some basic results from [12].

\section{Preliminaries}

Let $\Lambda$ be a positive integer such that all messages that we consider are of length at most $\Lambda$ bits. (Note that we used $L$ to denote the maximum number of elements of $\mathbb{F}$ that a message can consist of, so that, $\Lambda \geq L\left\lceil\log _{2} \mathbb{F}\right\rceil$.) 
A random (but, not necessarily uniform random) function $f:\{0,1\}^{\Lambda} \rightarrow\{0,1\}^{t}$ is said to have a collision bound of $\varepsilon$, if for distinct $x, x^{\prime} \in\{0,1\}^{\Lambda}, \operatorname{Pr}\left[f(x)=f\left(x^{\prime}\right)\right] \leq \varepsilon$. Such a function is said to be $\varepsilon$-almost universal ( $\varepsilon$-AU). The function $f$ is said to be $\varepsilon$-almost XOR universal ( $\varepsilon$-AXU) if for distinct $x, x^{\prime} \in\{0,1\}^{\Lambda}$ and any $\alpha \in\{0,1\}^{t}, \operatorname{Pr}\left[f(x) \oplus f\left(x^{\prime}\right)=\alpha\right] \leq \varepsilon$. A weaker notion of AU is to require that the collision bound holds only if the distinct strings $x, x^{\prime}$ have equal lengths. Let $\mathbb{F}$ be a finite field. Then a random function $f: \cup_{i=1}^{L} \mathbb{F}^{i} \rightarrow \mathbb{F}$ is defined to be $\varepsilon$-AU or $\varepsilon$-AXU in a manner similar to above.

Elements in the domain of $f$ are called messages and the value $f(x)$ is called the digest (or authenticator) of $x$ obtained using $f$. A random function $f$ can be realised from a function family $\left\{f_{K}\right\}_{K \in \mathcal{K}}$, where $\mathcal{K}$ is called the key space. A key $K$ is chosen uniformly at random from $\mathcal{K}$ and then $f$ is set to $f_{K}$. The randomness in $f$ comes from the random choice of $K$ and hence, the probabilities mentioned above are over the random choice of $K$.

For $\left(x_{1}, \ldots, x_{r}\right) \in \mathbb{F}^{r}$ define poly $\alpha\left(x_{1}, \ldots, x_{r}\right)=x_{r}+x_{r-1} \alpha+\cdots+x_{1} \alpha^{r-1}$. Using Horner's rule poly ${ }_{\alpha}\left(x_{r}, \ldots, x_{1}\right)$ can be evaluated using $(r-1)$ multiplications (over $\mathbb{F}$ ) and can be shown to be $(r-1) /|\mathbb{F}|$-AU for equal length messages. Further, $\alpha$ poly ${ }_{\alpha}\left(x_{1}, \ldots, x_{r}\right)$ is $r /|\mathbb{F}|$-AXU for equal length messages and can be evaluated using $r$ multiplications.

Bernstein [2] has introduced a class of polynomials which builds upon previous work due to Rabin and Winograd [8]. We call these the BRW polynomials. For $n \geq 2, \operatorname{BRW}_{\alpha}\left(x_{1}, \ldots, x_{r}\right)$ is $(2 r-1) /|\mathbb{F}|$-AU and can be evaluated using $\lfloor r / 2\rfloor$ multiplications and $\lg r$ squarings (to compute the powers $\left.\alpha^{2}, \alpha^{4}, \ldots\right)$. Further, $\alpha \operatorname{BRW}_{\alpha}\left(x_{1}, \ldots, x_{r}\right)$ is $2 r /|\mathbb{F}|$-AXU.

We will need another kind of AXU function. A random function $\psi:\{1, \ldots, r\} \rightarrow G F\left(2^{t}\right)$ is said to be $1 / 2^{t}$-AXU if for distinct $i, j$ with $1 \leq i, j \leq r$ and any $\alpha \in G F\left(2^{t}\right), \operatorname{Pr}[\psi(i) \oplus \psi(j)=\alpha]=1 / 2^{t}$.

Let $\tau(x)$ be a primitive polynomial of degree $t$ over $G F(2)$ and suppose that this $\tau(x)$ is used to define $G F\left(2^{t}\right)$. Let $\kappa$ be a uniform random element of $G F\left(2^{t}\right)$ and define $\psi: i \mapsto x^{i} \kappa \bmod \tau(x)$. The element $\kappa$ is the key to the function $\psi$. It is not difficult to show that such a $\psi$ satisfies $\frac{1}{2^{t}}$-AXU property if $r \leq 2^{t}-2$. A general definition of $\psi$ and a more efficient instantiation using word oriented LFSRs can be found in [10].

\section{The New Construction}

Let $\mathbb{F}$ be a finite field and $s$ and $t$ be integers such that $2^{s} \leq \# \mathbb{F} \leq 2^{t}$. Any $s$-bit element can be encoded into an element of $\mathbb{F}$ and any element of $\mathbb{F}$ can be encoded into a $t$-bit string. The values of $s$ and $t$ would depend on the choice of $\mathbb{F}$ and the representation of the elements of $\mathbb{F}$. If $\mathbb{F}$ is a binary extension field, then we can also have $s=t$ and $\mathbb{F}=G F\left(2^{t}\right)$. Given an $s$-bit string str, an injective function toElem encodes str into an element of $\mathbb{F}$; similarly, given an element $z \in \mathbb{F}$, an injective function toStr encodes $z$ into a $t$-bit string.

Messages to be hashed are bit strings of lengths greater than or equal to zero (and of maximum length $\Lambda$ bits). Given a message msg of length $n$ bits, an injective function pad maps msg $\mapsto$ $\operatorname{msg}\left\|0^{k}\right\| \operatorname{bin}_{s}(n)$, where $k$ is the minimum non-negative integer such that $n+k$ is a multiple of $s$ and $\operatorname{bin}_{s}(n)$ is the $s$-bit binary representation of $n$. Suppose $\operatorname{pad}(\operatorname{msg})=\operatorname{str}_{1}\|\cdots\| \operatorname{str}_{r}$ for some $r \geq 1$ and each $\operatorname{str}_{i}$ is a string of length $s$. The function parse $(\mathrm{msg})$ encodes each $\operatorname{str}_{i}$ into an element

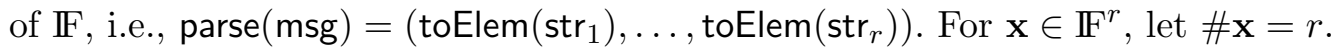

Let $m \geq 2$ be a fixed positive integer and let $\ell$ be an integer such that for any message msg, \#parse $(\mathrm{msg}) \leq m^{\ell} \triangleq L$. Let $f_{1}, \ldots, f_{\ell}$ be mutually independent random functions where each $f_{i}: D_{m} \rightarrow \mathbb{F}$ with $D_{m}=\cup_{i=1}^{m} \mathbb{F}^{i}$ having collision bound $\varepsilon_{i}$ for equal length messages. Here $\varepsilon_{i}$ could 
Fig. 1. Definition of the function reduce $(f, \mathbf{x})$.

reduce $(f, \mathbf{x})$ :
1. $\operatorname{parse~} \mathbf{x}$ as $\mathbf{x}=\left(\mathbf{x}_{1}, \ldots, \mathbf{x}_{k-1}, \mathbf{x}_{k}\right)$
$\quad$ where $\# \mathbf{x}_{i}=m$ for $1 \leq i \leq k-1$ and $1 \leq \# \mathbf{x}_{k} \leq m ;$
2. $\operatorname{return~}\left(f\left(\mathbf{x}_{1}\right), \ldots, f\left(\mathbf{x}_{k-1}\right), \ldots, f\left(\mathbf{x}_{k}\right)\right)$

Fig. 2. Definition of multi-level hashing.

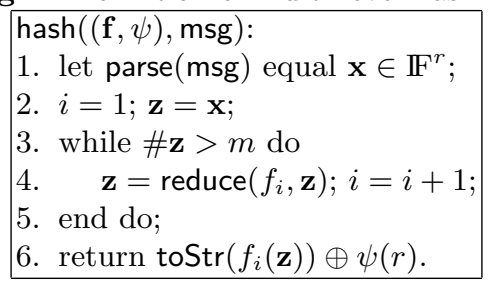

depend on $m$ and for polynomial hashing it indeed does. Note that $1 / 2^{t} \leq 1 / \# \mathbb{F} \leq \varepsilon_{i}$ for $1 \leq i \leq \ell$. We also need a random function $\psi:\{1, \ldots, \ell\} \rightarrow G F\left(2^{t}\right)$ such that for $1 \leq i, j \leq \ell$ with $i \neq j$, $\operatorname{Pr}[\psi(i) \oplus \psi(j)=\alpha]=1 / 2^{t}$ for any $\alpha \in G F\left(2^{t}\right)$. This $\psi$ is to be independent of $f_{1}, \ldots, f_{\ell}$. The functions $f_{1}, \ldots, f_{\ell}$ and $\psi$ can be instantiated as mentioned in Section 2 and we provide further discussion later.

Given a function $f: D_{m} \rightarrow \mathbb{F}$, the function reduce $(f, \mathbf{x})$ with $\mathbf{x} \in \mathbb{F}^{i}$ for some $i$, is defined in Figure 1. Using reduce and a sequence of functions $\mathbf{f}=\left(f_{1}, \ldots, f_{\ell}\right)$ as mentioned above, we define a hash function hash $((\mathbf{f}, \psi)$, msg) as given in Figure 2. The message msg to be hashed is a bit string of length greater than or equal to zero.

Note that reduce does not actually need to count the number of blocks in $\mathbf{x}$; the requirement is to divide $\mathbf{x}$ into $m$-element groups with the last group possibly having less than $m$ elements. Online processing is possible using the following strategy. Start the computation at the lowest level. After $m$ elements have been processed, one element of the next level is available for processing. Each group of $m$ elements at the bottom level gives rise to one element at the next level and hence, the processing at the next level can proceed in a synchronised manner with that of the bottom level. The rate at which this level processes will be $1 / m$ times the rate at which the bottom level processes. This is not particular to the bottom and the last-but-one level. The same strategy can be applied to higher levels, i.e., the processing at level $i$ proceeds at a rate of $1 / m$ of the processing at level $i-1$.

The requirement will be at most $\ell$ variables to store the intermediate values at the $\ell$ levels and additionally $\ell$ counters, one for each level, to keep track of the fact that a group of $m$ elements have been processed at that level. The counter for each level would have to be reset to 0 after every group of $m$ elements have been processed at that level. The memory requirement for processing a message having $r=$ \#parse(msg) is proportional to $2\left\lceil\log _{m} r\right\rceil$.

Theorem 1. Let msg, msg' be messages of lengths $n, n^{\prime} \geq 0$ such that

1. $\mathrm{msg} \neq \mathrm{msg}^{\prime}$,

2. $1 \leq \#$ parse $(\mathrm{msg})$, \#parse $\left(\mathrm{msg}^{\prime}\right) \leq m^{\ell}$, and

3. $y=\operatorname{hash}((\mathbf{f}, \psi), \mathrm{msg}), y^{\prime}=\operatorname{hash}((\mathbf{f}, \psi), \mathrm{msg})$.

Then $\operatorname{Pr}\left[y=y^{\prime}\right] \leq \varepsilon_{1}+\cdots+\varepsilon_{\ell}$ where $\varepsilon_{i}$ is the collision probability of $f_{i}$. 
Note. The requirement on $f_{i}$ is that they are $\mathrm{AU}$ for equal length messages, whereas the theorem states that hash is $\mathrm{AU}$ even for variable length messages.

Proof. Let $\mathbf{x}=$ parse(msg), $r=\# \mathbf{x}$ and $j$ be such that $m^{j-1} \leq r \leq m^{j}$. Then the functions $f_{1}, \ldots, f_{j}$ are used by hash on input msg. In the algorithm to compute hash, let $\mathbf{z}_{0}=\mathbf{x}$ and denote the output of reduce in the $i$ th iteration by $\mathbf{z}_{i}$, i.e.,

$$
\mathbf{x}=\mathbf{z}_{0}, \mathbf{z}_{1}=\operatorname{reduce}\left(f_{1}, m, \mathbf{z}_{0}\right), \ldots, \mathbf{z}_{j}=\operatorname{reduce}\left(f_{j}, m, \mathbf{z}_{j-1}\right) .
$$

The primed variables denote the similar quantities for $\mathrm{msg}^{\prime}$.

Case $r \neq r^{\prime}$. In this case,

$$
\begin{aligned}
\operatorname{Pr}\left[y=y^{\prime}\right] & =\operatorname{Pr}\left[\operatorname{toStr}\left(\mathbf{z}_{j}\right) \oplus \psi(r)=\operatorname{toS} \operatorname{tr}\left(\mathbf{z}_{j^{\prime}}^{\prime}\right) \oplus \psi\left(r^{\prime}\right)\right] \\
& =\operatorname{Pr}\left[\psi(r) \oplus \psi\left(r^{\prime}\right)=\operatorname{toS} \operatorname{tr}\left(\mathbf{z}_{j}\right) \oplus \operatorname{toStr}\left(\mathbf{z}_{j^{\prime}}^{\prime}\right)\right] \\
& \stackrel{(a)}{=} \frac{1}{2^{t}} \\
& \leq \frac{1}{|\mathbb{F}|} \leq \epsilon_{1}+\cdots+\epsilon_{\ell} .
\end{aligned}
$$

The step $(a)$ follows form the XOR universal property of $\psi$.

Case $r=r^{\prime}$. This implies that $j=j^{\prime}$ and from the definition of hash, $y=y^{\prime}$ if and only if $\mathbf{z}_{j}=\mathbf{z}_{j}^{\prime}$.

The lengths of $\operatorname{pad}(\mathrm{msg})$ and $\operatorname{pad}\left(\mathrm{msg}^{\prime}\right)$ are equal; if $n \neq n^{\prime}$, then the last $s$ bits of $\operatorname{pad}(\operatorname{msg})$ and $\operatorname{pad}\left(\mathrm{msg}^{\prime}\right)$ are not equal; if $n=n^{\prime}$, then the equal length messages msg and $\mathrm{msg}^{\prime}$ themselves are not equal. In both cases, we have $\mathbf{x} \neq \mathbf{x}^{\prime}$.

For $0 \leq i \leq j$, let $\mathrm{Eq}_{i}$ be the event $\mathbf{z}_{i}=\mathbf{z}_{i}^{\prime}$. We are interested in the event $\mathrm{Eq}_{j}$ and since $\mathbf{z}_{0}=$ $\mathbf{x} \neq \mathbf{x}^{\prime}=\mathbf{z}_{0}^{\prime}$, the probability of $\mathrm{Eq}_{0}$ is 0 . Write $\mathbf{z}_{i}=\left(\mathbf{z}_{i, 1}, \ldots, \mathbf{z}_{i, l_{i}}\right)$ with $\# \mathbf{z}_{i, k}=m$ for $1 \leq k \leq l_{i}-1$ and $1 \leq \# \mathbf{z}_{i, l_{i}} \leq m$. Then we can write $\mathbf{z}_{i+1}=\left(y_{i+1,1}, \ldots, y_{i+1, l_{i}}\right)$ where $y_{i+1, k}=f_{i+1}\left(\mathbf{z}_{i, k}\right)$. Since $r=r^{\prime}$, we have $l_{i}=l_{i}^{\prime}$ for $1 \leq i \leq \ell$. Now the event $\mathrm{Eq}_{i+1}$ can be written as $\bigwedge_{k=1}^{l_{i}}\left(y_{i+1, k}=y_{i+1, k}^{\prime}\right)$. The event $\overline{\mathrm{Eq}_{i}}$ means that $\left(\mathbf{z}_{i, 1}, \ldots, \mathbf{z}_{i, l_{i}}\right) \neq\left(\mathbf{z}_{i, 1}^{\prime}, \ldots, \mathbf{z}_{i, l_{i}}^{\prime}\right)$ so that for at least one $k, \mathbf{z}_{i, k} \neq \mathbf{z}_{i, k}^{\prime}$ and then for this $k, \operatorname{Pr}\left[f_{i+1}\left(\mathbf{z}_{i, k}\right)=f_{i+1}\left(\mathbf{z}_{i, k}^{\prime}\right)\right] \leq \varepsilon_{i+1}$ by the collision bound on $f_{i+1}$. So,

$$
\begin{aligned}
\operatorname{Pr}\left[\bigwedge_{k=1}^{l_{i}}\left(y_{i+1, k}=y_{i+1, k}^{\prime}\right) \mid \overline{\mathrm{Eq}_{i}}\right] & =\operatorname{Pr}\left[\bigwedge_{k=1}^{l_{i}}\left(f_{i+1}\left(\mathbf{z}_{i, k}\right)=f_{i+1}\left(\mathbf{z}_{i, k}^{\prime}\right)\right) \mid \overline{\mathrm{Eq}_{i}}\right] \\
& \leq \min _{1 \leq k \leq l_{i}} \operatorname{Pr}\left[f_{i+1}\left(\mathbf{z}_{i, k}\right)=f_{i+1}\left(\mathbf{z}_{i, k}^{\prime}\right) \mid \overline{\mathrm{Eq}_{i}}\right] \\
& \leq \varepsilon_{i+1} .
\end{aligned}
$$

For $0 \leq i \leq j-1$,

$$
\begin{aligned}
\operatorname{Pr}\left[\mathrm{Eq}_{i+1}\right]=\operatorname{Pr}\left[\mathbf{z}_{i+1}=\mathbf{z}_{i+1}^{\prime}\right] & =\operatorname{Pr}\left[\bigwedge_{k=1}^{l_{i}}\left(y_{i+1, k}=y_{i+1, k}^{\prime}\right)\right] \\
& =\operatorname{Pr}\left[\bigwedge_{k=1}^{l_{i}}\left(y_{i+1, k}=y_{i+1, k}^{\prime}\right) \mid \mathrm{Eq}_{i}\right] \operatorname{Pr}\left[\mathrm{Eq}_{i}\right]
\end{aligned}
$$




$$
\begin{aligned}
& +\operatorname{Pr}\left[\bigwedge_{k=1}^{l_{i}}\left(y_{i+1, k}=y_{i+1, k}^{\prime}\right) \mid \overline{\mathrm{Eq}_{i}}\right] \operatorname{Pr}\left[\overline{\mathrm{Eq}_{i}}\right] \\
= & \operatorname{Pr}\left[\mathrm{Eq}_{i}\right]+\operatorname{Pr}\left[\bigwedge_{k=1}^{l_{i}}\left(y_{i+1, k}=y_{i+1, k}^{\prime}\right) \mid \overline{\mathrm{Eq}_{i}}\right] \operatorname{Pr}\left[\overline{\mathrm{Eq}_{i}}\right] \\
\leq & \operatorname{Pr}\left[\mathrm{Eq}_{i}\right]+\operatorname{Pr}\left[\bigwedge_{k=1}^{l_{i}}\left(y_{i+1, k}=y_{i+1, k}^{\prime}\right) \mid \overline{\mathrm{Eq}_{i}}\right] \\
\leq & \operatorname{Pr}\left[\mathrm{Eq}_{i}\right]+\varepsilon_{i+1} .
\end{aligned}
$$

The last step follows from (1). Extending this we obtain

$$
\operatorname{Pr}\left[\mathrm{Eq}_{j}\right] \leq \operatorname{Pr}\left[\mathrm{Eq}_{j-1}\right]+\varepsilon_{j} \leq \cdots \leq \operatorname{Pr}\left[\mathrm{Eq}_{0}\right]+\varepsilon_{j}+\cdots+\varepsilon_{1} \leq \varepsilon_{j}+\cdots+\varepsilon_{1}
$$

XOR Universal. The hash function defined in Figure 2 provides almost universality. For certain applications, the requirement is to obtain XOR universality. It is possible to modify the algorithm to obtain XOR universality. Suppose each $f$ is instantiated using either poly or BRW. As mentioned earlier, both $\alpha$ poly $\alpha$ and $\alpha \mathrm{BRW}_{\alpha}$ are XOR universal. We refer to these variants by $f^{\mathrm{XOR}}$.

Suppose in algorithm hash, $m^{j-1}<r \leq m^{j}$. To obtain XOR universality, in Figure 2, the last line is changed to

$$
\text { return toStr }\left(f_{i}^{\mathrm{XOR}}(\mathbf{z})\right) \oplus \psi(r) .
$$

This means that the message is processed with $f_{1}, \ldots, f_{j-1}, f_{j}^{\mathrm{XOR}}$ instead of being processed with $f_{1}, \ldots, f_{j-1}, f_{j}$. In other words, for the first $(j-1)$ layers the usual functions are used, while for the last layer we use the variant which is XOR universal. It is not difficult to show that this achieves XOR universality of the entire construction.

\subsection{Instantiating $\psi$ and $\mathrm{f}=\left(f_{1}, \ldots, f_{\ell}\right)$}

Let $\alpha_{1}, \ldots, \alpha_{\ell}$ be independent and uniform random elements of $\mathbb{F}$. Further, let $\alpha$ be a uniform random element of $G F\left(2^{t}\right)$ which is independent of $\alpha_{1}, \ldots, \alpha_{\ell}$.

The key for the function $\psi$ is $\alpha$ and $f_{i}$ is instantiated as either poly $\alpha_{i}$ or as BRW $\alpha_{i}$. The collision bound and the number of multiplications required depend on the instantiations of $f_{i}$.

Case $f$ as poly. In this case, each $f_{i}$ has collision bound $(m-1) /|\mathbb{F}|$ and from Theorem 1 , the collision bound for hash $((\mathbf{f}, \psi)$, msg $)$ is $\ell(m-1) /|\mathbb{F}| \leq m\left\lceil\log _{m} L\right\rceil /|\mathbb{F}|$ where any message consists of at most $L$ elements of $\mathbb{F}$. The key consists of $1+\left\lceil\log _{m} L\right\rceil$ elements of $\mathbb{F}$. The number of multiplications required by hash to process msg with \#parse $(\mathrm{msg})=r$ equals $r-1$.

Case $f$ as BRW. Using Theorem 1 , the collision bound is $\ell(2 m-1) /|\mathbb{F}|$ and the key size is the same as for poly. The number of multiplications required to process msg with parse $(\mathrm{msg})=r$ is at most equal to

$$
\frac{n}{2} \times\left(\frac{m^{j+1}-1}{m^{j}(m-1)}\right)=\frac{n}{2} \times\left(1+\frac{1}{m-1}\left(\frac{m^{j}-1}{m^{j}}\right)\right) .
$$


Here $j$ is the unique positive integer such that $m^{j-1}<r \leq m^{j}$.

Let $n_{0}=n$ and $n_{i}=\left\lceil n_{i-1} / m\right\rceil$ for $0<i \leq s$ with $n_{s}=1$. The number of multiplications equals

$$
\left\lfloor\frac{m}{2}\right\rfloor \sum_{i=1}^{j}\left(n_{i}-1\right)+\left\lfloor\frac{r_{1}}{2}\right\rfloor+\cdots+\left\lfloor\frac{r_{s}}{2}\right\rfloor \leq\left\lfloor\frac{n_{0}}{2}\right\rfloor+\left\lfloor\frac{n_{1}}{2}\right\rfloor+\cdots+\left\lfloor\frac{n_{s-1}}{2}\right\rfloor .
$$

If $n_{0}=m^{j}$ for some $j$, then the number of multiplications required is $\frac{m}{2}\left(\frac{m^{j}-1}{m-1}\right)$.

Combination of poly and BRW. It is possible to combine the two options, i.e., instantiate some of the $f$ 's using poly and the other f's using BRW. One reason for doing this could be the issue of pre-computation. The computation of poly ${ }_{\alpha}$ can be speeded up using a pre-computed table based on $\alpha$ and this cannot be done for $\mathrm{BRW}_{\alpha}$. So, one option is to use poly to instantiate $f_{1}$ while $f_{2}, \ldots, f_{\ell}$ are instantiated using BRW. Since most of the multiplications are done at the lowest level, the pre-computed table can be used to speed-up these multiplications. Further, keeping multiplication tables for each $\alpha_{i}$ can be costly in terms of storage and so one can use BRW hashing for the higher levels since this requires lesser number of multiplications.

\section{References}

1. Daniel J. Bernstein. The Poly1305-AES message-authentication code. In Henri Gilbert and Helena Handschuh, editors, FSE, volume 3557 of Lecture Notes in Computer Science, pages 32-49. Springer, 2005.

2. Daniel J. Bernstein. Polynomial evaluation and message authentication, 2007. http://cr.yp.to/papers.html\# pema.

3. Jürgen Bierbrauer, Thomas Johansson, Gregory Kabatianskii, and Ben J. M. Smeets. On families of hash functions via geometric codes and concatenation. In Douglas R. Stinson, editor, CRYPTO, volume 773 of Lecture Notes in Computer Science, pages 331-342. Springer, 1993.

4. John Black, Shai Halevi, Hugo Krawczyk, Ted Krovetz, and Phillip Rogaway. UMAC: Fast and secure message authentication. In Michael J. Wiener, editor, CRYPTO, volume 1666 of Lecture Notes in Computer Science, pages 216-233. Springer, 1999.

5. Larry Carter and Mark N. Wegman. Universal classes of hash functions. J. Comput. Syst. Sci., 18(2):143-154, 1979.

6. Edgar N. Gilbert, F. Jessie MacWilliams, and Neil J. A. Sloane. Codes which detect deception. Bell System Technical Journal, 53:405-424, 1974.

7. Shai Halevi and Hugo Krawczyk. MMH: Software message authentication in the gbit/second rates. In Eli Biham, editor, Fast Software Encryption, volume 1267 of Lecture Notes in Computer Science, pages 172-189. Springer, 1997.

8. Michael O. Rabin and Shmuel Winograd. Fast evaluation of polynomials by rational preparation. Communications on Pure and Applied Mathematics, 25:433-458, 1972.

9. Phillip Rogaway. Bucket hashing and its application to fast message authentication. J. Cryptology, 12(2):91-115, 1999.

10. Palash Sarkar. A general mixing strategy for the ECB-Mix-ECB mode of operation. Inf. Process. Lett., 109(2):121-123, 2008.

11. Victor Shoup. On fast and provably secure message authentication based on universal hashing. In Neal Koblitz, editor, CRYPTO, volume 1109 of Lecture Notes in Computer Science, pages 313-328. Springer, 1996.

12. Douglas R. Stinson. Universal hashing and authentication codes. Des. Codes Cryptography, 4(4):369-380, 1994.

13. Mark N. Wegman and Larry Carter. New hash functions and their use in authentication and set equality. J. Comput. Syst. Sci., 22(3):265-279, 1981. 